\title{
Bienestar psicológico y orientación de rol sexual en estudiantes universitarios
}

\section{Psychological well-being and sex role orientation in university students}

\author{
Enrique Barra \\ Universidad de Concepción, Chile \\ (Rec: 01 abril 2009 / Acep: 31 marzo 2010)
}

\begin{abstract}
Resumen
Este estudio examinó la relación entre el bienestar psicológico, las dimensiones de masculinidad y feminidad y las categorías de orientación de rol sexual (masculino, femenino, andrógino, indiferenciado). Los participantes fueron 346 estudiantes de ambos sexos de la Universidad de Concepción (Chile) con edades entre 18 y 31 años, quienes respondieron instrumentos referentes a bienestar psicológico y dimensiones de rol sexual. Los resultados mostraron que el bienestar psicológico presentaba mayor relación con la dimensión de masculinidad que con la de feminidad, y que los participantes categorizados como indiferenciados presentaron un nivel significativamente menor de bienestar psicológico que el resto de las orientaciones de rol sexual, siendo los individuos andróginos los que informaron el mayor nivel de bienestar. Se proponen diversos factores para explicar los resultados obtenidos.

Palabras claves: orientación de rol sexual; bienestar psicológico; masculinidad; feminidad.
\end{abstract}

\begin{abstract}
This study was designed to estimate the relationships between psychological well-being, masculinity, femininity and the sex role orientation categories (masculine, feminine, androgynous, undifferentiated). Participants were 346 male and female students, 18 to 31 years old, from the University de Concepción (Chile). Individuals completed measures of psychological well-being and sex role orientation. Results show that psychological well-being had higher relationships with masculinity than with femininity and those participants with undifferentiated orientation reported lower well-being scores than did those with other orientations, being the androgynous category associated with the highest reported well-being. Diverse explanatory factors for obtained results are proposed.
\end{abstract}

Key words: sex role orientation; psychological well-being; masculinity; femininity. 


\section{Introducción}

Al igual como sucede con muchas otras dimensiones psicológicas, existen distintos enfoques para conceptualizar y evaluar el bienestar individual (Kim-Prieto, Diener, Tamir, Scollon \& Diener, 2005). Esta diversidad de aproximaciones al concepto de bienestar se debería a que este constructo puede abarcar un amplio rango de componentes tanto cognitivos como afectivos y referirse a distintos aspectos, entre ellos calidad de vida, felicidad, satisfacción con la vida, balance afectivo y también ausencia de estrés o depresión.

Diversos autores (Deci \& Ryan, 2008; Peterson, Park \& Seligman, 2005; Samman, 2007) distinguen dos grandes perspectivas en el estudio del bienestar: el enfoque hedónico centrado en la felicidad y que define el bienestar en términos de obtención de placer y evitación del dolor, y el enfoque eudaimónico centrado en la autorrealización que define el bienestar en términos de desarrollo del potencial humano y funcionamiento psicológico pleno. Respecto a estos grandes enfoques se ha planteado que la perspectiva hedónica ha sido representada durante mucho tiempo por el concepto de bienestar subjetivo, mientras que el enfoque eudaimónico estaría mejor representado por el concepto más reciente de bienestar psicológico (Keyes, Shmotkin \& Ryff, 2002; Ryff \& Singer, 2008). Mientras que el bienestar subjetivo (hedónico) se refiere a la felicidad, satisfacción vital y balance afectivo, el bienestar psicológico (eudaimónico) se refiere a la evaluación del individuo de su desarrollo personal (Keyes, 2006; Ring, Höfer, McGee, Hickey \& O’Boyle, 2007).

Integrando diversos conceptos de la tradición eudaimónica en psicología de la personalidad, del desarrollo y clínica, Ryff (1989) formuló un modelo multidimensional del bienestar psicológico conformado por seis factores, que incluyen la evaluación positiva de uno mismo y la propia vida pasada (autoaceptación); un sentido de crecimiento continuo y desarrollo como persona (crecimiento personal); la creencia de que la propia vida tiene un propósito y un significado (propósito en la vida); el desarrollo y mantenimiento de relaciones de calidad y confianza con otros (relaciones positivas con otros); la capacidad de dirigir efectivamente la propia vida y el ambiente para satisfacer necesidades y deseos (dominio ambiental); y un sentido de autodeterminación personal (autonomía). Cada una de estas dimensiones indica los desafíos que enfrentan los individuos en sus intentos por funcionar plenamente y realizar sus potencialidades (Keyes, 2006).

$\mathrm{Al}$ evaluar estas dimensiones del bienestar psicológico en una muestra de adultos estadounidenses de distintas edades, Ryff (1989) encontró diferencias en algunos aspectos del bienestar psicológico en relación con algunas variables como edad, nivel educacional y género. Respecto al género, la única diferencia significativa era que las mujeres mostraban mayor nivel que los hombres en la dimensión de relaciones positivas con otros. En cambio en un estudio con adultos suecos entre 32 y 58 años, Lindfors, Berntsson \& Lundberg (2006) encontraron que además de la diferencia de género señalada, las mujeres también presentaban mayor nivel en la dimensión de propósito en la vida y los hombres presentaban mayor nivel que las mujeres en la dimensión de dominio ambiental.

El presente estudio se propuso examinar en estudiantes universitarios las posibles diferencias de género en el bienestar psicológico y además analizar la relación entre los indicadores de bienestar y la orientación de rol sexual de los individuos.

La investigación acerca de la orientación de rol sexual puede adoptar una perspectiva continua-dimensional o bien una perspectiva categórica. La perspectiva continua involucra medir el grado de masculinidad y el grado de feminidad de cada individuo, ya sea hombre o mujer, para establecer la relación de cada una de estas dimensiones por separado con otros factores o variables. Por su parte la perspectiva categórica implica clasificar a los individuos en categorías de orientación de rol sexual de acuerdo a sus niveles relativos en las dimensiones de masculinidad y de feminidad (Bem, 1975). Si un individuo, ya sea hombre o mujer, obtiene altos puntajes de masculinidad y de feminidad se categoriza como andrógino; si tiene un alto grado de masculinidad y un bajo grado de feminidad se categoriza como masculino; si su nivel de masculinidad es bajo y su nivel de feminidad es alto se categoriza como femenino; por último si obtiene bajos puntajes tanto de masculinidad como de feminidad se categoriza como indiferenciado.

Las escasas evidencias existentes acerca de la relación entre bienestar y orientación de rol sexual muestran resultados inconsistentes, lo cual podría explicarse en parte por la utilización de formas muy diferentes de evaluar el bienestar. Así por ejemplo, mientras September, McCarrey, Baranowsky, Parent y Schindler (2001) midieron el bienestar mediante las dimensiones de bienestar psicológico de Ryff falta el año descritas anteriormente, Johnson et al., (2006) utilizaron como indicadores de bienestar medidas de soledad, incomodidad personal, autoestima, ansiedad social y evitación social. Y además los resultados difieren según se utilice el criterio continuo o el categórico para evaluar la orientación de rol sexual.

Respecto a las medidas continuas de la orientación de rol sexual, September et al., (2001) informaron que la dimensión de masculinidad presentaba correlaciones más altas que la dimensión de feminidad con las distintas subescalas de bienestar psicológico de Ryff falta el año, excepto en la subescala de relaciones positivas con otros que mostraba mayor relación con feminidad. Y respecto a las categorías de orientación de rol sexual, estos autores encontraron que las categorías de andrógino y femenino obtenían puntajes superiores en la subescala de relaciones positivas con otros respecto a las categorías de masculino e indiferenciado, 
mientras que las categorías de andrógino y masculino obtenían puntajes superiores en la subescala de autonomía respecto a las categorías de femenino e indiferenciado.

Por su parte, y utilizando medidas más indirectas de bienestar, Johnson y otros (2006) encontraron que las categorías de andrógino y masculino se asociaban a mayores niveles de bienestar que las categorías de femenino e indiferenciado, mientras que respecto a las medidas continuas de las dimensiones de rol sexual se encontró que los indicadores de bienestar mostraban una relación positiva con la masculinidad y una relación negativa con la feminidad.

Considerando la escasez de evidencias empíricas, especialmente en nuestro medio, el presente estudio tuvo como objetivos:

1. Determinar la relación existente entre el bienestar psicológico y las dimensiones de masculinidad y feminidad.

2. Examinar posibles diferencias en bienestar psicológico entre los individuos con distintas categorías de orientación de rol sexual.

3. Examinar posibles diferencias de género en el bienestar psicológico y en sus distintas dimensiones.

\section{Método}

\section{Participantes}

La muestra estuvo conformada por 346 estudiantes (159 hombres y 187 mujeres) de la Universidad de Concepción (Chile), con edades entre 18 y 31 años (promedio de 20.6 años). Los estudiantes estaban cursando desde primer a tercer año de 14 carreras de las áreas matemática, biológica, humanidades y ciencias sociales.

\section{Instrumentos}

Bienestar psicológico: Se utilizó la Escala de Bienestar Psicológico de Ryff (1989), destinada a evaluar la percepción de bienestar personal en seis dimensiones: autoaceptación ("Me gusta mi forma de ser"), relaciones positivas con otros ("Mantener relaciones cercanas ha sido difícil y frustrante para mi"), crecimiento personal ("Para mí, la vida ha sido un continuo proceso de aprendizaje, cambio y crecimiento"), propósitos en la vida ("A veces me cuesta encontrar sentido a las cosas que hago"), dominio ambiental ("Soy capaz de manejar adecuadamente las demandas de la vida diaria") y autonomía ("Soy muy influenciable por la opinión de otros"). Cada dimensión incluye 3 ítems, con un formato de respuesta tipo Likert de cuatro puntos, desde "completamente de acuerdo" a "completamente en desacuerdo". En este estudio se obtuvieron índices de consistencia interna mediante coeficiente Alpha de 0.79 para las dimensiones de autoaceptación, dominio ambiental y autonomía, de 0.80 para relaciones positivas con otros, de 0.78 para crecimiento personal y de 0.73 para autonomía.

Rol sexual: Se utilizó el Inventario de Rol Sexual elaborado y validado en Chile por Barra (2002, 2004), destinado a evaluar en qué medida los individuos perciben en sí mismo ciertas características masculinas (instrumentales) y femeninas (expresivas). Consiste en 30 ítems, 15 de los cuales corresponden a la escala de Masculinidad (M) y 15 a la escala de Feminidad (F), con un formato de respuesta tipo Likert de cinco puntos (desde "nunca o casi nunca" a "siempre o casi siempre"). En su elaboración se obtuvieron índices de consistencia interna mediante coeficiente Alpha de 0.82 para $\mathrm{M}$ y de 0.84 para $\mathrm{F}$ y una estabilidad temporal de 0.70 para $\mathrm{M}$ y de 0.72 para $\mathrm{F}$ con intervalo de un año. Respecto a su validez de constructo, la escala M presentó correlaciones positivas significativas con motivación de logro, asertividad y control emocional, y negativas con motivación de afiliación, empatía y sensibilidad social. En cambio la escala $\mathrm{F}$ correlacionó positivamente con empatía, sensibilidad emocional, expresividad emocional, sensibilidad social y motivación de afiliación, mientras que correlacionó negativamente con control emocional y motivación de logro (Barra, 2004). En el presente estudio se obtuvieron índices de consistencia interna mediante coeficiente Alpha de 0.74 para $\mathrm{M}$ y de 0.75 para F.

\section{Procedimiento}

Los instrumentos fueron aplicados en forma colectiva y anónima durante horas de clases. Luego de obtener la autorización de los docentes de las asignaturas, se solicitó la participación voluntaria de los alumnos, enfatizando el carácter anónimo de la información recolectada.

\section{Resultados}

Para cumplir con el primer objetivo se calcularon los coeficientes de correlación de Pearson entre los indicadores de bienestar psicológico y las dimensiones de masculinidad y feminidad. En la Tabla 1 se puede observar que las dimensiones de autoaceptación, dominio ambiental y autonomía muestran una relación notoriamente mayor con la masculinidad que con la feminidad, tanto en hombres como en mujeres. En cambio la dimensión de relaciones con otros presenta mayor relación con la feminidad que con la masculinidad, especialmente en las mujeres. Respecto al segundo objetivo, examinar diferencias en bienestar entre las distintas orientaciones de rol sexual, se agrupó a los participantes en las cuatro orientaciones de acuerdo a su posición respecto a las medianas de los puntajes en las escalas de masculinidad y feminidad. Ya que la mediana de ambas escalas fue de 53 puntos, los puntajes iguales o superiores a 54 fueron catalogados como altos y aquellos iguales o inferiores a 52 fueron catalogados como bajos. 
Tabla 1. Correlaciones entre los indicadores de bienestar y las dimensiones de masculinidad (M) y feminidad (F).

\begin{tabular}{lrrrrrr} 
& \multicolumn{2}{c}{ Total } & \multicolumn{2}{c}{ Mombres } & \multicolumn{2}{c}{ Mujeres } \\
\hline Variables & $\mathrm{M}$ & $\mathrm{F}$ & $\mathrm{M}$ & $\mathrm{F}$ & $\mathrm{M}$ & $\mathrm{F}$ \\
\hline Bienestar Psicológico global & $0.35^{* *}$ & $0.25^{* *}$ & $0.46^{* *}$ & $0.35^{* *}$ & $0.26^{* *}$ & $0.15^{*}$ \\
Autoaceptación & $0.26^{* *}$ & 0.10 & $0.32^{* *}$ & 0.15 & $0.20^{* *}$ & 0.08 \\
Dominio Ambiental & $0.35^{* *}$ & $0.11^{*}$ & $0.40^{* *}$ & $0.18^{*}$ & $0.29^{* *}$ & 0.09 \\
Propósito en la Vida & $0.12^{*}$ & $0.19^{* *}$ & $0.18^{*}$ & $0.23^{* *}$ & 0.08 & 0.09 \\
Relaciones con Otros & $0.22^{* *}$ & $0.42^{* *}$ & $0.37^{* *}$ & $0.52^{* *}$ & 0.09 & $0.33^{* *}$ \\
Crecimiento Personal & $0.14^{* *}$ & $0.13^{*}$ & $0.24^{* *}$ & 0.14 & 0.06 & 0.10 \\
Autonomía & $0.33^{* *}$ & 0.00 & $0.33^{* *}$ & 0.13 & $0.33^{* *}$ & -0.09 \\
\hline p $<0.05{ }^{* *} \mathrm{p}<0.01$ & & & & & &
\end{tabular}

De acuerdo a esto, cuando un individuo presentaba puntajes altos en ambas escalas se categorizaba como Andrógino, si los puntajes eran bajos en ambas escalas era Indiferenciado, si presentaba puntaje alto en feminidad y bajo en masculinidad se consideraba Femenino y cuando el puntaje era alto en masculinidad y bajo en feminidad era categorizado como Masculino. Como esta categorización excluyó a aquellos participantes que tenían uno o ambos puntajes justo en la mediana, para este análisis la muestra se redujo de 346 a 314 participantes.

Mediante un análisis de varianza unifactorial se constató la existencia de diferencias significativas en todas las dimensiones de bienestar psicológico entre las distintas categorías de orientación de rol sexual, realizándose la prueba de comparaciones múltiples post-hoc de Bonferroni para determinar cuáles categorías eran las que diferían significativamente entre sí (Tabla 2). El análisis mostró que los indiferenciados presentaban un nivel significativamente inferior de bienestar psicológico global que las otras tres categorías, observándose este mismo patrón en cuanto a las dimensiones de autoaceptación y de crecimiento personal. Los andróginos y masculinos presentaban un nivel significativamente mayor de dominio ambiental que los femeninos e indiferenciados, mientras que los andróginos y femeninos presentaban un nivel significativamente mayor de propósito en la vida que los indiferenciados y de relaciones con otros que los masculinos e indiferenciados. Por último, respecto a la dimensión de autonomía, los masculinos y andróginos presentaban un nivel significativamente mayor que los indiferenciados y además los masculinos presentaban mayor nivel que los femeninos.

En cuanto al tercer objetivo, examinar posibles diferencias de género en las diversas variables, la prueba t de diferencia de medias reveló que sólo existían diferencias significativas, a favor de las mujeres, en la dimensión de propósito en la vida del bienestar psicológico y en la escala de feminidad. Un resultado muy destacable fue que en la dimensión de masculinidad no se observó una diferencia de género significativa (Tabla 3).

\section{Discusión}

Las principales conclusiones del presente estudio son (a) en general los indicadores de bienestar mostraron mayores relaciones con la masculinidad que con la feminidad; (b) los indiferenciados informaron menor nivel de bienestar psicológico que las otras categorías de orientación de rol sexual, mientras que los andróginos eran los que presentaban en general mayores niveles de bienestar; y (c) se observó una diferencia significativa de género sólo en una de las seis dimensiones del bienestar psicológico.

En relación con la primera conclusión, los resultados concuerdan con la literatura que en general informa una mayor relación entre masculinidad y reporte de bienestar, que entre éste y feminidad (Barret \& White, 2002; September et al., 2001; Ward, 2000). A diferencia del estudio de Johnson et al., (2006), quienes encontraron que el bienestar estaba asociado positivamente con la masculinidad y negativamente con la feminidad, en el presente estudio se encontraron relaciones positivas entre los indicadores de bienestar y la feminidad, aunque en general de magnitud inferior a las existentes entre bienestar y masculinidad. Una de las razones de esta discrepancia puede ser que, a diferencia del presente estudio que utilizó medidas específicas de bienestar, en el estudio de Johnson et al., (2006) el bienestar se evaluaba de manera más indirecta, mediante mediciones de constructos tales como soledad, autoestima $\mathrm{y}$ ansiedad social. 
Tabla 2. Medias y desviaciones estándar de los indicadores de bienestar en las categorías de andróginos (A), masculinos (M), femeninos (F) e indiferenciados (I).

\begin{tabular}{|c|c|c|c|c|}
\hline Variables & $\begin{array}{c}\mathrm{A} \\
(\mathrm{n}=75)\end{array}$ & $\begin{array}{c}\mathrm{M} \\
(\mathrm{n}=75)\end{array}$ & $\begin{array}{c}\mathrm{F} \\
(\mathrm{n}=84)\end{array}$ & $\begin{array}{c}\mathrm{I} \\
(\mathrm{n}=80)\end{array}$ \\
\hline Bienestar Psicológico global & $58.2(7.1) \mathrm{a}$ & $56.7(5.6) \mathrm{a}$ & $56.1(4.7) \mathrm{a}$ & $50.8(7.5) \mathrm{b}$ \\
\hline Autoaceptación & $9.9(1.7) \mathrm{a}$ & $9.9(1.5) \mathrm{a}$ & $9.6(1.4) \mathrm{a}$ & $8.9(1.7) b$ \\
\hline Dominio Ambiental & $9.1(1.4) \mathrm{a}$ & $9.0(1.5) \mathrm{a}$ & $8.2(1.2) \mathrm{b}$ & $7.6(1.9) \mathrm{b}$ \\
\hline Propósito en la Vida & $9.6(1.9) \mathrm{a}$ & $9.0(1.7)$ & $9.4(1.3) \mathrm{a}$ & $8.5(1.6) \mathrm{b}$ \\
\hline Relaciones con Otros & $9.9(1.7) \mathrm{a}$ & $8.6(1.9) b$ & $9.6(1.6) \mathrm{a}$ & $7.9(1.7) b$ \\
\hline Crecimiento Personal & $10.1(1.7) \mathrm{a}$ & $9.9(1.7) \mathrm{a}$ & $9.9(1.2) \mathrm{a}$ & $9.1(1.9) \mathrm{b}$ \\
\hline Autonomía & $9.7(1.7) \mathrm{ab}$ & $10.2(1.4) \mathrm{a}$ & $9.4(1.5) b c$ & $8.7(1.9) \mathrm{c}$ \\
\hline
\end{tabular}

Nota. Las medias en la misma fila con distintos subíndices son significativamente diferentes al nivel de 0.05 , de acuerdo al análisis de comparaciones múltiples post hoc de Bonferroni.

Tabla 3. Diferencias entre hombres y mujeres en las diversas variables

\begin{tabular}{|c|c|c|c|c|c|}
\hline \multirow[b]{2}{*}{ Variable } & \multicolumn{2}{|c|}{ Hombres (N=159) } & \multicolumn{2}{|c|}{ Mujeres (N=187) } & \multirow{2}{*}{$\mathrm{t}$} \\
\hline & Media & D.S & Media & D.S & \\
\hline Bienestar Psicológico global & 55.45 & 6.93 & 55.75 & 6.81 & 0.40 \\
\hline Autoaceptación & 9.66 & 1.68 & 9.58 & 1.60 & 0.44 \\
\hline Dominio Ambiental & 8.57 & 1.68 & 8.37 & 1.59 & 1.12 \\
\hline Propósito en la Vida & 8.94 & 1.77 & 9.35 & 1.64 & $2.23 *$ \\
\hline Relaciones con Otros & 8.99 & 1.90 & 9.17 & 1.80 & 0.86 \\
\hline Crecimiento Personal & 9.69 & 1.63 & 9.84 & 1.69 & 0.89 \\
\hline Autonomía & 9.60 & 1.66 & 9.43 & 1.72 & 0.93 \\
\hline Masculinidad & 53.21 & 8.21 & 52.33 & 8.03 & 1.00 \\
\hline Feminidad & 49.42 & 10.47 & 55.88 & 8.89 & $6.20 * * *$ \\
\hline
\end{tabular}

Respecto a la segunda conclusión, el hecho que los individuos indiferenciados presenten niveles significativamente menores de bienestar psicológico que el resto de las orientaciones de rol sexual, podría explicarse porque tendrían menos capacidad de adaptación a diversas demandas debido al menor desarrollo de algunos recursos psicológicos característicos de las dimensiones de masculinidad y feminidad. Por el contrario, los individuos andróginos mostrarían mayores niveles de bienestar porque la adecuada adaptación psicológica de un individuo dependería de su posesión de características tanto masculinas (o instrumentales) como femeninas (o expresivas), ya que de esa manera tendrían mayor capacidad para comportarse de modo flexible según lo requieran las circunstancias (Bem, 1975). Esto se reflejaría en que los individuos andróginos obtuvieron los mayores puntajes tanto en el bienestar psicológico global como en sus diversas dimensiones, con la excepción de autonomía, en la cual obtuvieron mayor puntaje los individuos masculinos. 
En cuanto a las otras dos categorías de orientación de rol sexual, los individuos masculinos tienden a presentar puntajes algo mayores que los femeninos en la mayoría de las dimensiones de bienestar psicológico, excepto en las dimensiones de propósito en la vida y relaciones positivas con otros. Estos resultados son consistentes con lo informado por Johnson y otros (2006), quienes encontraron que los individuos masculinos mostraban mayor nivel de bienestar que los femeninos. De acuerdo a Hoffmann, Powlishta \& White (2004), esta diferencia se relaciona con el hecho que la masculinidad se asocia a la posesión de rasgos instrumentales, mientras que la feminidad se asocia a la posesión de rasgos expresivos. Los rasgos instrumentales promueven el ajuste y el bienestar facilitando el equilibrio afectivo que sirve como amortiguador del estrés, haciendo que los individuos valoren los eventos estresantes como menos amenazantes y adopten estrategias más activas de afrontamiento. En cambio los rasgos expresivos, más orientados hacia lo interpersonal y lo emocional, pueden afectar negativamente el ajuste y el bienestar haciendo que el individuo experimente y exprese más sus emociones negativas. Esto sería especialmente aplicable a los estudiantes universitarios, considerando que el ambiente académico promueve la posesión y despliegue de rasgos instrumentales al mismo tiempo que minimiza la importancia de los rasgos expresivos, por lo cual los individuos que poseen más rasgos instrumentales tendrían potencialmente mayor capacidad de adaptación a ese ambiente.

Respecto a la tercera conclusión de este estudio, sólo se encontró una diferencia de género significativa en la dimensión de propósito en la vida, en la cual las mujeres presentaron mayor puntaje que los hombres, lo cual concuerda con lo encontrado por Vleioras \& Bosma (2005) en universitarios griegos y por Lindfors et al., (2006) en trabajadores suecos. Sin embargo, en ambos estudios también se encontraron diferencias a favor de las mujeres en la dimensión de relaciones positivas con otros, una diferencia que en el presente estudio no fue estadísticamente significativa.

Un resultado adicional interesante fue que mientras se encontró una diferencia de género altamente significativa en la dimensión de feminidad a favor de las mujeres, no se observó una diferencia de género significativa en masculinidad, en la cual había sólo una muy ligera diferencia a favor de los hombres. A pesar de lo inesperado, este resultado es similar al encontrado por September et al., (2001) en universitarios canadienses, y puede ser interpretado a la luz de una tendencia señalada por diversos autores (Auster \& Ohm, 2000; Twenge, 1997), en el sentido que a través del tiempo se observa una progresiva disminución en las diferencias de género en la dimensión de masculinidad, la cual se explicaría porque los puntajes de masculinidad de las mujeres se han ido incrementando con el transcurso del tiempo por una progresiva mayor valorización social de tales características. Al comparar los resultados del presente estudio con los obtenidos en el estudio de validación del instrumento de rol sexual en otra muestra de la misma población de estudiantes universitarios (Barra, 2004), se constata que la tendencia señalada ya se observa en nuestro medio, ya que el principal cambio observado entre ambas muestras en el transcurso de pocos años es el aumento en el promedio de masculinidad de las mujeres.

El hecho de que no se observen diferencias significativas de género en el bienestar psicológico y en la mayoría de sus dimensiones, y que al mismo tiempo existan diferencias significativas en tales dimensiones entre las distintas orientaciones de rol sexual, permitiría concluir que el bienestar psicológico de los individuos no estaría determinado primariamente por su pertenencia a uno u otro sexo, sino que por la integración de características tanto instrumentales como expresivas y el desarrollo de sus diversas potencialidades humanas.

Se sugiere para futuras investigaciones incluir participantes con un rango más amplio de edad con el fin de examinar posibles cambios con la edad en las relaciones entre el bienestar psicológico y las variables de género, así como examinar la influencia de otras variables, como el nivel educacional, el nivel socioeconómico y el estado civil. También sería deseable investigar las relaciones entre el bienestar psicológico y otras características personales como la autoeficacia, la percepción de apoyo social, los estilos de afrontamiento del estrés, los estilos cognitivos, los estilos de apego, etc. Mientras más conocimiento se tenga de los diversos factores que se relacionan con el bienestar personal, se estará en mejores condiciones para promover y desarrollar aquellas condiciones que posibilitan una mayor satisfacción consigo mismo y con la propia vida.

\section{Referencias}

Auster, C., \& Ohm, S. (2000). Masculinity and femininity in contemporary American society: A reevaluation using the Bem Sex-Role Inventory. Sex Roles, 43, 499-528

Barra, E. (2002). Construcción de un inventario de rol sexual en universitarios chilenos. Revista Intercontinental de Psicología y Educación, 4, 11-19.

Barra, E. (2004). Validación de un inventario de rol sexual construido en Chile. Revista Latinoamericana de Psicología, 36, 97-106.

Barret, A., \& White, H. (2002). Trajectories of gender role orientations in adolescence and early adulthood: A prospective study of the mental health effects of masculinity and femininity. Journal of Health and Social Behavior, 43, 451-468.

Bem, S. (1975). Sex role adaptability: One consequence of psychological androgyny. Journal of Personality and Social Psychology, 31, 634-643.

Deci, E., \& Ryan, R. (2008). Hedonia, eudaimonia, and well-being: An introduction. Journal of Happiness Studies, 9, 1-11.,

Hoffmann, M., Powlishta, K., \& White, K. (2004). An examination of gender differences in adolescent adjustment: The effect of competence on gender role differences in symptoms of psychopathology. Sex Roles, 50, 795-810. 
Johnson, D., McNair, R., Vojick, A., Congdon, D., Monacelli, J., \& Lamont, J. (2006). Categorical and continuous measurement of sex-role orientation: Differences in associations with young adults' reports of well being. Social Behavior and Personality, 34, 59-76.

Keyes, C. (2006). Subjective well-being in mental health and human development research worldwide: An introduction. Social Indicators Research, 77, 1-10.

Keyes, C.; Shmotkin, D., \& Ryff, C. (2002). Optimizing well-being: The empirical encounter of two traditions. Journal of Personality and Social Psychology, 82, 1007-1022.

Kim-Prieto, Ch., Diener, E., Tamir, M., Scollon, C., \& Diener, M. (2005). Integrating the diverse definitions of happiness: A time-sequential framework of subjective well-being. Journal of Happiness Studies, 6, 261-300.

Lindfors, P., Berntsson, L., \& Lundberg, U. (2006). Factor structure of Ryff's psychological well-being scales in Swedish female and male white-collar workers. Personality and Individual Differences, 40, 1213-1222.

Peterson, C., Park, N., \& Seligman, M. (2005). Orientations to happiness and life satisfaction: The full life versus the empty life. Journal of Happiness Studies, 6, 25-41.

Ring, L., Höfer, S., McGee, H., Hickey, A., \& O’Boyle, C. (2007). Individual quality of life: Can it be accounted for by psychological or subjective well-being? Social Indicators Research, 82, 443-461.
Ryff, C. D. (1989). Happiness is everything, or is it? Explorations on the meaning of psychological well-being. Journal of Personality and Social Psychology, 57, 1069-1081.

Ryff, C., \& Singer, B. (2008). Know thyself and become what you are: A eudaimonic approach to psychological well-being. Journal of Happiness Studies, 9, 13-39.

Samman, E. (2007). Psychological and subjective well-being: A proposal of internationally comparable indicators. Oxford Development Studies, $35,459-486$.

September, A., McCarrey, M., Baranowsky, A., Parent, Ch., \& Schindler, D. (2001). The relation between well-being, impostor feelings, and gender role orientation among Canadian university students. The Journal of Social Psychology, 141, 218-232.

Twenge, J. (1997). Changes in masculine and feminine traits over time: A meta-analysis. Sex Roles, 36, 305-325.

Vleioras, G., \& Bosma, H. (2005). Are identity styles important for psychological well-being? Journal of Adolescence, 28, 397-409.

Ward, C. (2000). Models and measurements of psychological androgyny: A cross-cultural extension of theory and research. Sex Roles, 43, 529552. 